\title{
Comparison of Low Level Laser and Arginine-Calcium Carbonate Alone or Combination in the Treatment of Dentin Hypersensitivity: A Randomized Split-Mouth Clinical Study
}

\author{
Mehmet Vehbi Bal, DDS, PhD, illker Keskiner, DDS, PhD, ${ }^{2}$ Ufuk Sezer, DDS, PhD, ${ }^{3}$ \\ Cengizhan Açıkel, MD, PhD, ${ }^{4}$ and Işll Saygun, DDS, $\mathrm{PhD}^{1}$
}

\begin{abstract}
Objective: This study aimed to compare the efficacy of low-level laser (LLL) and desensitizing paste (DP) containing $8 \%$ arginine-calcium carbonate, in the treatment of dentin hypersensitivity (DH) and also to determine whether their combined application would improve the efficacy of the treatment. Background data: There are various options for the treatment of DH; however, superiority of one method over others alone has not been currently demonstrated. Materials and methods: Twenty-one patients with 156 teeth affected by DH were included in the study. Selected teeth were randomly divided into five groups: LLL, DP, laser followed by DP $(\mathrm{LLL}+\mathrm{DP}), \mathrm{DP}$ followed by laser (DP + LLL) applied to one of the quadrants, and a control group, consisting of a randomly selected additional tooth in one of the quadrants. Teeth were irradiated by the $685 \mathrm{~nm}$ diode laser treatment with $25 \mathrm{~mW}$ at $9 \mathrm{~Hz}$ for $100 \mathrm{sec}$ at $1 \mathrm{~cm}^{2}$ area $\left(2 \mathrm{~J} / \mathrm{cm}^{2}\right)$ in interrupted mode. Pain response to evaporative stimulus was quantified on a visual analogue scale (VAS) over a 90-day period. Results: All four treatment groups experienced significant and persistent decrease in the mean VAS score immediately posttreatment until the end of the study, whereas the placebo group had high VAS scores throughout the study. On day 90, percent reduction in VAS scores was $72 \%$ for LLL, $65.4 \%$ for DP, $54.6 \%$ for LLL + DP, and $69.6 \%$ for DP + LLL, whereas the placebo group showed an increase of 7.8\%. Conclusions: The application of either LLL or DP containing $8 \%$ arginine-calcium carbonate appears to be effective in decreasing DH. However, their combined use does not improve the efficacy beyond what is attainable with either treatment alone.
\end{abstract}

\section{Introduction}

D ENTIN HYPERSENSITIVITY (DH) IS AN ACUTE, sharp pain in exposed dentin, experienced in response to various stimuli such as thermal changes, occlusal forces, an acidic diet, brushing, and evaporative stimulus, which cannot be explained by any other dental defect or pathology. ${ }^{1,2}$ Etiologic factors for $\mathrm{DH}$ include gingival recession and enamel loss, both conditions resulting in exposure of underlying dentin. ${ }^{2}$ Overzealous tooth brushing and surgical or nonsurgical treatment of periodontal disease are predisposing factors for gingival recession, whereas enamel loss may be related to tooth wear by attrition, erosion, or abrasion. ${ }^{2}$ The mechanism of DH is generally explained by the hydrody- namic theory, which states that external stimuli cause fluid displacement within the dentin tubules, leading to compression or stretching in the outermost odontoblasts within the pulp, thereby triggering nerve endings and evoking the feeling of pain. ${ }^{3}$ Therefore, DH treatment mainly involves restriction of fluid movement within dentin tubules and/or inhibition of pulpal nerve response.

Low and moderate level lasers encompassing a wide range of wavelengths have been used in the treatment of $\mathrm{DH}$ with variable success. Low-level lasers (LLL) such as He-Ne, emitting visible spectrum red light at $630 \mathrm{~nm}$, or GaAlAs lasers at 780,830 , or $900 \mathrm{~nm}$, are thought to act by increasing the action potential of the nerve cells, thereby limiting the transmission of pain stimulus. ${ }^{4}$ LLL do not modify the morphology

\footnotetext{
${ }^{1}$ Department of Periodontology, Dental Sciences Center, Gulhane Military Medical Academy, Ankara, Turkey.

${ }^{2}$ Department of Periodontology, Faculty of Dentistry, Ondokuz Mays University, Samsun, Turkey.

${ }^{3}$ Department of Periodontology, Faculty of Dentistry, Bezmialem Vakif University, Istanbul, Turkey.

${ }^{4}$ Department of Public Health, Gulhane Military Medical Academy, Ankara, Turkey.
} 
of dentin tubules, whereas moderate-level lasers such as NdYAG, emitting infrared light at $1064 \mathrm{~nm}$, stimulate secondary dentin production and seal open tubules, as well as having an immediate analgesic effect on nerve cells. ${ }^{4}$

In addition to laser treatments, various topical agents such as fluorides, potassium oxalate, strontium chloride, and silver nitrate have been tested, and they have shown variable efficacy and duration of effect. ${ }^{5}$ Recent research indicated that certain naturally occurring components of the saliva have desensitizing properties that provide DH relief. Based on the composition of saliva, a new topical agent was developed, containing $8 \%$ arginine in bicarbonate buffer along with calcium carbonate. ${ }^{6}$ When applied to exposed dentin, this composition forms a plug containing arginine, calcium, phosphate, and carbonate in dentin tubules, and reduces DH by inhibiting fluid movement within tubules. ${ }^{6}$

In addition to the individual effects of laser and topical agents, their combined use has been shown to have additive or synergistic effects in the treatment of DH. Combination of $\mathrm{Nd}$ :YAG laser irradiation with sodium fluoride varnish was reported to have a synergistic effect on human dential tubules. ${ }^{7}$ Furthermore, Omae et al. have shown that Er:YAG irradiation increased bond strength of densensitizing agents containing glutaraldehyde and HEMA. ${ }^{8}$ When used in combination, laser irradiation is usually generally applied following the use of topical agents, a technique that has been shown to cover dentin canals. ${ }^{9-11}$ However, the effect of initial laser irradiation followed by the application of topical paste containing $8 \%$ arginine-calcium carbonate has not been studied thus far. Furthermore, although previous studies prove that desensitizing paste (DP) containing $8 \%$ arginine-calcium carbonate had higher efficacy than other local agents in treating $\mathrm{DH}$, its efficacy has not been evaluated in comparison with or in combination with laser irradiation, particularly LLL.

It is hypothesized that the preapplication of laser irradiation may increase the efficacy of DP containing $8 \%$ argininecalcium carbonate. Therefore, in this study, the aim was to compare the efficacy of LLL and DP containing $8 \%$ argininecalcium carbonate in teeth with DH. Moreover, it was also aimed to determine whether their combined use would improve the efficacy of DH treatment through an additive or synergistic effect.

\section{Materials and Methods}

\section{Patient selection}

Patients who were admitted to the periodontology department of Gülhane Military Medical Academy (GMMA) with com- plaints of DH were invited to participate in the study. Twentyone patients of similar sociocultural background [16 women, 5 men; mean age $37 \pm 12$ (range, 19-60) years] having a total of 156 teeth with confirmed DH were included. The inclusion criteria were as follows: being 19-60 years of age, being in good systemic health, having two or more teeth showing hypersensitivity to air blast in each of the four quadrants, good oral hygiene, and agreeing to a 3-month follow-up. The exclusion criteria were as follows: benign or malignant pathological oral lesions, chronic disease, chronic medication use, caries in selected or neighboring teeth, cracked enamel, orthodontic appliance, restorations, congenital enamel and/or dentin defects, history of vital bleaching, periodontal disease, periodontal surgery or hypersensitivity treatment within the past 6 months, nonsurgical treatment of periodontal disease within the past 3 months, use of dentifrice or mouthwash containing a desensitizing agent, being pregnant or lactating, use of antidepressants or analgesics, and allergy to any of the contents of the treatment.

All patients were thoroughly informed about the treatment plan, possibilities of discomfort, and risks. Written informed consent was obtained from all participants. Study protocol and consent forms were approved by the Institutional Ethics Committee of Gülhane Military Medical Academy (protocol number: 10-1539).

\section{Treatment protocol}

A split-mouth study design was adopted because of the advantages of there being the same pain perception, oral hygiene habits, dietary habits, and psychosomatic factors. Each patient's mouth was divided into four quadrants, and different applications were performed to each quadrant. After giving oral hygiene education at the first examination, the four mouth quadrants of each patient were randomized to apply one of the four study treatments. A randomly selected tooth in one of the quadrants was defined as the control. In each patient, a randomly selected quadrant was given one session of one of the following treatments: LLL; DP containing 8\% argininecalcium carbonate; LLL followed by DP containing $8 \%$ arginine-calcium carbonate (LLL + DP); and DP containing $8 \%$ arginine-calcium carbonate followed by LLL (DP + LLL). The control tooth was given physiological saline solution (placebo). Thus, all teeth in the same quadrant received the same treatment except for the control tooth. The treatment allocation according to the types of teeth is presented in Table 1.

Laser treatment was administered using a diode laser (BTL2000 Laser, Dravotnicka Techika, Prague, Czech Republic) applied to the vestibule of the affected tooth interrupted for

Table 1. Treatment Allocation According to the Types of Teeth

\begin{tabular}{lcccccrr}
\hline & $\begin{array}{c}\text { Maxillary } \\
\text { molars }\end{array}$ & $\begin{array}{c}\text { Mandibular } \\
\text { molars }\end{array}$ & $\begin{array}{c}\text { Maxillary } \\
\text { premolars }\end{array}$ & $\begin{array}{c}\text { Mandibular } \\
\text { premolars }\end{array}$ & $\begin{array}{c}\text { Maxillary } \\
\text { anterior }\end{array}$ & $\begin{array}{c}\text { Mandibular } \\
\text { anterior }\end{array}$ & Total \\
\hline LLL & $3(7.3)$ & $2(4.9)$ & $8(19.5)$ & $9(22)$ & $5(12.2)$ & $14(34.1)$ & $41(100)$ \\
DP & $2(6.3)$ & $1(3.1)$ & $8(25)$ & $9(28.1)$ & $8(25)$ & $4(12.5)$ & $32(100)$ \\
LLL+DP & $2(6.9)$ & $1(3.4)$ & $6(20.7)$ & $5(17.2)$ & $8(27.6)$ & $7(24.1)$ & $29(100)$ \\
DP+LLL & $3(9.4)$ & $0(-)$ & $6(18.8)$ & $10(31.3)$ & $3(9.4)$ & $10(31.3)$ & $32(100)$ \\
Placebo & $1(4.5)$ & $0(-)$ & $3(13.6)$ & $7(31.8)$ & $8(36.4)$ & $3(13.6)$ & $22(100)$ \\
Total & $11(7.1)$ & $4(2.6)$ & $31(19.9)$ & $40(25.6)$ & $32(20.5)$ & $38(24.4)$ & $156(100)$ \\
\hline
\end{tabular}

LLL, laser treatment; DP, desensitizing paste containing $8 \%$ arginine-calcium carbonate; LLL + DP, laser treatment followed by desensitizing paste; DP + LLL, desensitizing paste treatment followed by laser. 
$100 \mathrm{sec}$ at $685 \mathrm{~nm}$ wavelength with $25 \mathrm{~mW}$ power, $9 \mathrm{~Hz}$ frequency, and $2.0 \mathrm{~J} / \mathrm{cm}^{2}$ density at $1 \mathrm{~cm}^{2}$ area. ${ }^{12}$ The laser was applied with the optic fiber tip at a distance of $2 \mathrm{~mm}$ from the dental outer surface.

DP containing 8\% arginine-calcium carbonate (Colgate Sensitive Pro-Relif, Colgate-Palmolive Company, New York, NY) was applied using a rotating rubber cup (2000 rev/min) in two consecutive rounds lasting $3 \mathrm{sec}$ each.

On the first visit, teeth with $\mathrm{DH}$ were established, and all patients received oral hygiene training. A soft toothbrush and toothpaste without any antihypersensitivity agent were provided for use during the length of the study. In addition, subjects were asked not to use any mouthwash or fluoride products during the study period. The treatment was administered on the following visit. DH evaluation was performed pretreatment, immediately post-treatment, and on days $10,30,60$, and 90 post-treatment.

\section{Evaluation}

Hypersensitivity was tested by evaporative stimulus, which consisted of a short air blast (temperature range of $19^{\circ}-20^{\circ} \mathrm{C}$ ) applied perpendicularly to the root surface of the affected tooth using a periodontal probe (Williams periodontal probe, Kohdent-Kohler, Germany, 3106). To ensure the standardization of the distance between the tip of the root surface and air blast in patients with symptomatic $\mathrm{DH}$, the air blast was applied with the periodontal probe for $1 \mathrm{sec}$ from a distance of $1 \mathrm{~cm}$ without touching the root surface. Neighboring teeth were isolated using cotton rolls and a suction device to prevent false responses. All hypersensitivity tests were applied in the morning, in a quiet calm environment, by the same clinician using the same dental chair and equipment, with the same pressure (60 psi) and temperature air blast. After each stimulus, patients were asked to mark the intensity of pain on a $100 \mathrm{~mm}$ visual analogue scale (VAS), which was marked "No pain" at the left end and "Unbearable pain" at the right end.

In addition, the plaque index (PI) "Silness \& Löe"13 and gingival index (GI) "Löe \& Silness", 14 was applied. The bleeding on probing (BOP) score was recorded, and the probing depth (PD) and clinical attachment level (CAL) were measured. All measurements were performed with a periodontal probe from all four sides (mesial, distal, lingual, and buccal) of the tooth.

\section{Statistical analysis}

Statistical analysis was performed using PASW Statistics 18 (SPSS Inc., Chicago, IL). Data were expressed using descriptive statistics (mean, standard deviation, median, minimum, maximum, and percentage). The KolmogorovSmirnov test was used to test for the normality of distribution of clinical parameters measured at different periods. Because the significance level of the Kolmogorov-Smirnov test was $<0.05$, nonparametric tests were used for the comparison of study groups. The treatment groups were compared using the Kruskal-Wallis and Bonferronicorrected Mann-Whitney $U$ test (post-hoc). The Bonferronicorrected Wilcoxon signed ranks test was used for the comparison of repeated measurements within the same treatment group. The level of significance was determined to be $p<0.05$. To prevent the multiplicity problem, and to not

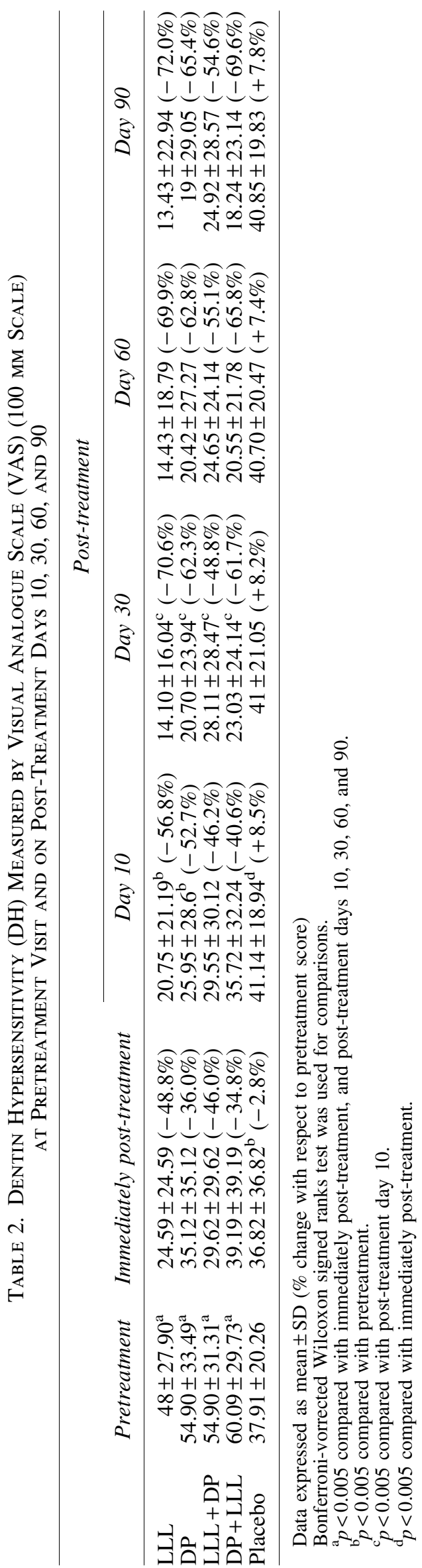


FIG. 1. Mean visual analogue scale (VAS) score during the study for each treatment group. LLL, laser treatment; DP, desensitizing paste containing $8 \%$ argininecalcium carbonate; LLL + DP, laser treatment followed by desensitizing paste; DP + LLL, desensitizing paste treatment followed by laser.

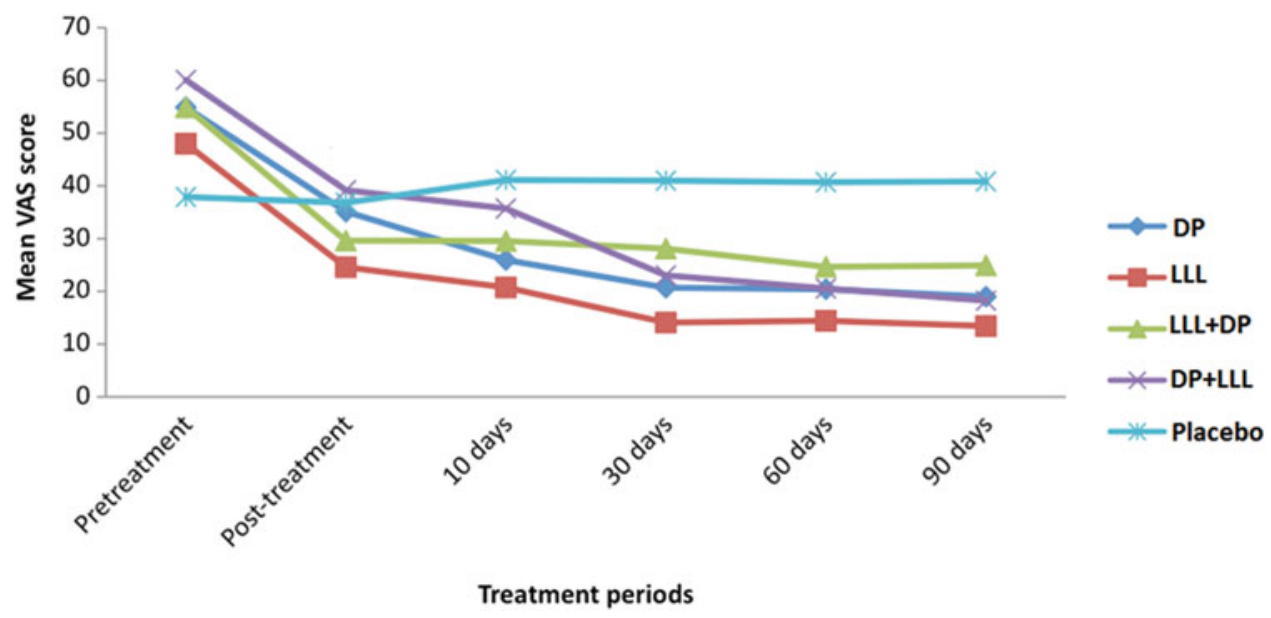

increase the predefined family-wise error rate, the Bonferronicorrected Mann-Whitney $U$ test was used as the post-hoc test. For the Bonferroni-corrected test, the statistical level of significance was defined as $<0.05 / 10=0.005$.

\section{Results}

All the patients completed the 90-day study period. The pretreatment mean VAS scores were not significantly different among the treatment groups (Kruskal-Wallis test, $p=0.085$ ). All four active treatment groups (LLL, DP, LLL + DP, and DP + LLL) experienced significant reduction in the mean VAS score immediately post-treatment, whereas the placebo group showed no significant difference. On day 30 , the mean VAS scores were significantly reduced compared with those on day 10 in all the active treatment groups, but not in the placebo group (Table 2 and Fig. 1). Improvement in $\mathrm{DH}$ was maintained until the end of the study on day 90 in all the active treatment groups. Compared with the placebo group, a significant reduction in the mean VAS score was observed on day 10 for the LLL and DP groups, on day 30 for the DP+LLL group, and on day 60 for the LLL, DP, LLL + DP, and DP + LLL groups (Bonferroni-corrected Wilcoxon signed ranks test, $p<0.005)$. The mean VAS scores of the LLL, DP, LLL + $\mathrm{DP}$, and DP + LLL groups were not significantly different at any time point. On day 90 , the percent reduction in VAS scores was $72 \%$ for LLL, $65.4 \%$ for DP, $54.6 \%$ for LLL+ $\mathrm{DP}$, and $69.6 \%$ for DP + LLL, whereas the placebo group showed an increase of $7.8 \%$ (Bonferroni-corrected Wilcoxon signed ranks test, $p<0.005$ ) (Table 2).

Oral hygiene, gingival inflammation, and recession were assessed during the course of the study. Pretreatment values of PI $(p=0.619)$, GI $(p=0.430)$, BOP $(p=0.455)$, PD $(p=0.441)$, and CAL $(p=0.859)$ were similar in all the active treatment groups and the placebo group (KruskalWallis test) (Table 3). No clinically significant changes were observed in any of the measurements during the course of the study. No side effects related to DH treatments were observed.

\section{Discussion}

In this study, one session of LLL or DP containing $8 \%$ arginine-calcium carbonate showed a comparable longlasting reduction in DH. However, no further benefit was gained by use of these two treatments in combination.

The in-office application of arginine-calcium carbonate has been evaluated in a number of randomized controlled clinical studies. Previous clinical trials using argininecalcium carbonate paste have showed that in patients with hypersensitive teeth, a single application of this paste following dental cleaning and scaling procedures provided instant relief lasting at least 28 days. ${ }^{6,13-15}$ Application of arginine-calcium carbonate paste before dental cleaning was similarly effective in reducing sensitivity to evaporative and tactile stimuli immediately after the cleaning procedure. ${ }^{16}$ In vitro analysis of treated teeth using confocal, atomic force,

Table 3. Pretreatment Measurements of Oral Hygiene and Periodontal Health of Teeth With Dentin Hypersensitivity (DH)

\begin{tabular}{lccccc}
\hline & $L L L$ & $D P$ & $L L L+D P$ & $D P+L L L$ & Placebo \\
\hline PI & $0.31 \pm 0.50$ & $0.29 \pm 0.33$ & $0.19 \pm 0.25$ & $0.20 \pm 0.21$ & $0.18 \pm 0.25$ \\
GI & $0.23 \pm 0.29$ & $0.41 \pm 0.43$ & $0.39 \pm 0.47$ & $0.41 \pm 0.47$ & $0.27 \pm 0.31$ \\
BOP (\%) & $5.47 \pm 10.50$ & $11.59 \pm 16.86$ & $11.38 \pm 18.80$ & $15.63 \pm 22.67$ & $7.95 \pm 11.92$ \\
PD (mm) & $1.66 \pm 0.44$ & $1.79 \pm 0.77$ & $1.81 \pm 0.61$ & $1.57 \pm 0.39$ & $1.77 \pm 0.51$ \\
CAL (mm) & $2.13 \pm 0.85$ & $2.22 \pm 1.05$ & $2.06 \pm 0.85$ & $1.96 \pm 0.99$ & $2.00 \pm 0.69$ \\
\hline
\end{tabular}

Data expressed as mean $\pm \mathrm{SD}$.

LLL, laser treatment; DP, desensitizing paste containing $8 \%$ arginine-calcium carbonate; LLL+DP, laser treatment followed by desensitizing paste; DP + LLL, desensitizing paste treatment followed by laser; PI, plaque index; GI, gingival index; BOP, bleeding on probing; PD, probing depth; CAL, clinical attachment level. 
and scanning electron microscopy confirmed that argininecalcium carbonate acts by plug formation and occlusion of dentin tubules. Moreover, significant reduction of dentin fluid flow was shown by hydraulic conductance analysis. ${ }^{17}$

More recently, independent clinical studies also confirmed these findings. In a 12-week, randomized, double-blind, splitmouth design clinical trial, the in-office application of arginine-calcium carbonate paste provided significant reduction in sensitivity compared with that of the calcium carbonate control, lasting during the entire follow-up period. ${ }^{18}$ In addition, in a randomized double-blind active control clinical trial, in-office treatment with arginine-calcium carbonate paste resulted in a significant reduction in sensitivity compared with sodium fluoride gel, which persisted during the 28 day follow-up period. Using scanning electron microscopy, studies also showed significantly better occlusion of tubules after arginine-calcium carbonate treatment compared with treatment with sodium fluoride gel. ${ }^{19}$ The percent reduction in sensitivity ranged between $45 \%$ and $60 \%$ in all of the above studies; therefore, the findings of the present study are consistent with the existing literature.

Various low- and moderate-level lasers have been tested for the treatment of DH. Even within the LLL classification, a wide variety of lasers, both in type and in wavelength, were used, from HeNe lasers emitting at $630 \mathrm{~nm}$ to GaAlAs lasers emitting at $900 \mathrm{~nm}$. Because the effect of lasers depends upon the wavelength of emitted light, the studies most pertinent to the present work were those employing lasers within the visible red spectrum.

The previous studies reported a significant reduction in sensitivity with respect to baseline using 660-685 $\mathrm{nm}$ diode lasers; however, their results were weakened by either the absence of placebo control or the lack of a significant difference with respect to the placebo. In studies of a $660 \mathrm{~nm}$ diode laser versus sodium fluoride ${ }^{20}$ and a $660 \mathrm{~nm}$ diode laser versus an $830 \mathrm{~nm}$ diode laser, ${ }^{21} \mathrm{DH}$ was similarly improved with respect to baseline in both treatment groups. In a study comparing Nd:YAG and $685 \mathrm{~nm}$ lasers, significant improvement was seen in both groups, although significantly greater reduction of sensitivity was reported for the $\mathrm{Nd}$ :YAG group at 60 days. ${ }^{12}$ Unfortunately, these studies lacked a placebo control group to verify the effect of active treatment, whereas two other studies employing a placebo control showed no difference compared with that of the placebo. In a study comparing a $670 \mathrm{~nm}$ laser with a placebo light over 6 weeks following six sessions of treatment, a significant reduction from the baseline was reported in both groups, with no significant difference observed between laser treatment and placebo. ${ }^{22}$ More recently, Vieira et al. ${ }^{23}$ compared four sessions of $660 \mathrm{~nm}$ laser, 3\% potassium oxalate gel, and placebo gel, and showed a similar reduction in sensitivity immediately and after 3 months of follow-up in all study groups, including the placebo group. In contrast, the present study suggests that a $685 \mathrm{~nm}$ diode laser has a significant desensitizing effect that is different from the placebo. and comparable to arginine-calcium carbonate paste.

A synergistic desensitizing effect was reported when sodium fluoride varnish was used in combination with the Nd:YAG laser. ${ }^{9,10}$ Because both treatments act by dentin tubule occlusion, their effect in combination was thought to be related to a higher sodium fluoride gel adhesion following laser treatment. In addition to this study, Lopes et al. ${ }^{11}$ performed a study using a low-power laser and a desensitizing agent, Gluma, separately and in combination, for a duration of 6 months, and they noted that all of the treatment options, including desensitizing methods, were effective with different mechanisms. In addition, the combination therapy seemed to be an effective alternative regimen in the treatment of DH. In a recent in vitro study, Tunar et al. ${ }^{24}$ assessed the effects of Er:YAG laser, desensitizing paste (DP) and their combinations on human dentine tubules utilizing scanning electron microscopic analysis. They used 40 freshly extracted teeth and divided them into four groups. Their first group was control, while group II, group III, and group IV received Er:YAG laser $(30 \mathrm{~Hz}, 60 \mathrm{~mJ} / \mathrm{pulse}, 10$ $\mathrm{sec}$ ), a DP containing $8 \%$ arginine and calcium carbonate, and DP + Er:YAG laser in combination, respectively. They noted that there was a statistically significant occlusion in dentine specimens in combination (DP + Er:YAG laser) group. However, in this study, there were no significant differences between the Er:YAG laser and DP and Er:YAG laser and combination groups, whereas a significant difference was found between the DP and combination groups. The result of this study, which is inconsistent with the present study, was thought to be conflicting. No synergistic effect was observed between a $685 \mathrm{~nm}$ diode LLL and arginine-calcium carbonate paste in the present study, perhaps because of the different mechanisms of action of these treatments.

Administering identical treatments to all groups so that the DP group received the placebo laser treatment, the LLL group received placebo DP application, and the placebo group received placebo laser and placebo DP treatments would have strengthened the present results; therefore, this constitutes a limitation of this study. Other limitations of the study that should be noted were the small sample size and the short follow-up time (not more than 90 days).

\section{Conclusions}

The following conclusions were reached.

- One-time application of either a $685 \mathrm{~nm}$ LLL, or DP containing $8 \%$ arginine-calcium carbonate, reduced $\mathrm{DH}$ both immediately and over the long term was performed without any adverse reactions.

- There were no additive or synergistic effects on sensitivity reduction in both treatments with combinations.

- To evaluate the efficacy of combined treatments with laser and paste and to establish a standard treatment for dentinal hypersensitivity, further prospective longitudinal studies should be performed with larger populations.

\section{Author Disclosure Statement}

No competing financial interests exist.

\section{References}

1. Addy M, Urquhart E. Dentine hypersensitivity: its prevalence, aetiology and clinical management. Dent Update 1992;19:407-408.

2. Dababneh RH, Khouri AT, Addy M. Dentine hypersensitivityan enigma? A review of terminology, mechanisms, aetiology and management. Br Dent J 1999;11:606-611. 
3. Brannstrom M, Astrom A. The hydrodynamics of the dentine; its possible relationship to dentinal pain. Int Dent $\mathbf{J}$ 1972;2:219-227.

4. Kimura Y, Wilder-Smith P, Yonaga K, Matsumoto K. Treatment of dentine hypersensitivity by lasers: a review. J Clin Periodontol 2000;10:715-721.

5. Matesanz P, Herrera D, Echeverría A, O'Connor A, González I, Sanz M. Current management of dentin hypersensitivity. Clin Oral Invest Suppl 2013;1:55-66.

6. Kleinberg I. SensiStat. A new saliva-based composition for simple and effective treatment of dentinal sensitivity pain. Dent Today 2002;12:42-47.

7. Lan WH, Liu HC, Lin CP. The combined occluding effect of sodium fluoride varnish and Nd:YAG laser irradiation on human dentinal tubules. J Endod 1999;25:424-426.

8. Omae M, Inoue M, Itota $\mathrm{T}$, et al. Effect of a desensitizing agent containing glutaraldehyde and HEMA on bond strength to Er:YAG laser-irradiated dentine. J Dent 2007;35:398-402.

9. Kumar NG, Mehta DS. Short-term assessment of the Nd:YAG laser with and without sodium fluoride varnish in the treatment of dentin hypersensitivity - a clinical and scanning electron microscopy study. J Periodontol 2005;7: 1140-1147.

10. Umberto R, Claudia R, Gaspare P, Gianluca T, Alessandro del V. Treatment of dentine hypersensitivity by diode laser: a clinical study. Int J Dent 2012;2012:858950.

11. Lopes AO, de Paula EC, Aranha AC. Clinical evaluation of low-power laser and a desensitizing agent on dentin hypersensitivity. Lasers Med Sci 2015 [Epub ahead of print].

12. Dilsiz A, Canakci V, Ozdemir A, Kaya Y. Clinical evaluation of Nd:YAG and 685-nm diode laser therapy for desensitization of teeth with gingival recession. Photomed Laser Surg 2009;6:843-848.

13. Silness J, Löe H. Periodontal disease in pregnancy. II. Correlation between oral hygiene and periodontal condition. Acta Odontol Scand 1964;22:121-135.

14. Löe H, Silness J. Periodontal disease in pregnancy. I. Prevalance and severity. Acta Odontol Scand 1963;21:533-551.

15. Schiff T, Delgado E, Zhang YP, Cummins D, DeVizio W, Mateo LR. Clinical evaluation of the efficacy of an inoffice desensitizing paste containing $8 \%$ arginine and calcium carbonate in providing instant and lasting relief of dentin hypersensitivity. Am J Dent 2009;22 Spec No A: $8 \mathrm{~A}-15 \mathrm{~A}$.

16. Hamlin D, Williams KP, Delgado E, Zhang YP, DeVizio W, Mateo LR. Clinical evaluation of the efficacy of a desensitizing paste containing $8 \%$ arginine and calcium carbonate for the in-office relief of dentin hypersensitivity associated with dental prophylaxis. Am J Dent 2009;22: 16A-20A.

17. Petrou I, Heu R, Stranick M, et al. A breakthrough therapy for dentin hypersensitivity: how dental products containing $8 \%$ arginine and calcium carbonate work to deliver effective relief of sensitive teeth. J Clin Dent 2009;1:23-31.

18. Kapferer I, Pflug C, Kisielewsky I, Giesinger J, Beier US, Dumfahrt H. Instant dentin hypersensitivity relief of a single topical application of an in-office desensitizing paste containing $8 \%$ arginine and calcium carbonate: a splitmouth, randomized-controlled study. Acta Odontol Scand 2013;3-4:994-999.

19. Uraz A, Erol-Simsek O, Pehlivan S, Suludere Z, Bal B. The efficacy of $8 \%$ arginine-CaCO3 applications on dentine hypersensitivity following periodontal therapy: a clinical and scanning electron microscopic study. Med Oral Patol Oral Cir Bucal 2013;2:e298-e305.

20. Corona SA, Nascimento TN, Catirse AB, Lizarelli RF, Dinelli W, Palma-Dibb RG. Clinical evaluation of low-level laser therapy and fluoride varnish for treating cervical dentinal hypersensitivity. J Oral Rehabil 2003;12:1183-1189.

21. Ladalardo TC, Pinheiro A, Campos RA, et al. Laser therapy in the treatment of dentine hypersensitivity. Braz Dent $\mathbf{J}$ 2004;2:144-150.

22. Gentile LC, Greghi SL. Clinical evaluation of dentin hypersensitivity treatment with the low intensity GalliumAluminum-Arsenide laser-AsGaAl. J Appl Oral Sci 2004; 4:267-272.

23. Vieira AH, Passos VF, de Assis JS, Mendonca JS, Santiago SL. Clinical evaluation of a $3 \%$ potassium oxalate gel and a GaAlAs laser for the treatment of dentinal hypersensitivity. Photomed Laser Surg 2009;5:807-812.

24. Tunar OL, Gürsoy H, Cakar G, Kuru B, Ipci SD, Yılmaz S. Evaluation of the effects of Er:YAG laser and desensitizing paste containing $8 \%$ arginine and calcium carbonate, and their combinations on human dentine tubules: a scanning electron microscopic analysis. Photomed Laser Surg 2014;32:540-545.

Address correspondence to:

Mehmet Vehbi Bal

Gulhane Military Medical Academy Dental Sciences Center

Department of Periodontology Etlik Caddesi, Ankara, 06018 Turkey

E-mail: vehbibal@hotmail.com 